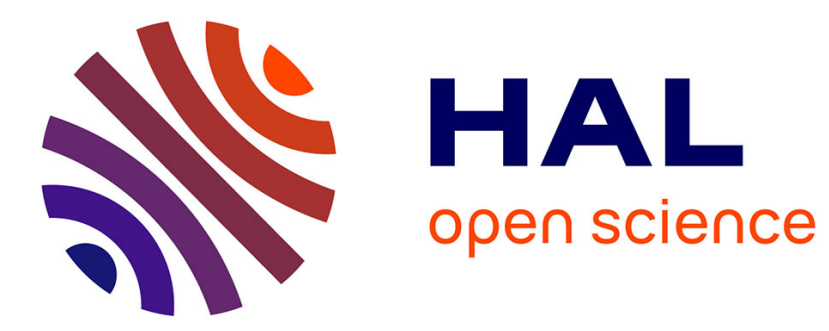

\title{
Spectral EEG-based classification for operator dyads' workload and cooperation level estimation
}

Kevin J. Verdière, Frédéric Dehais, Raphaëlle N. Roy

\section{To cite this version:}

Kevin J. Verdière, Frédéric Dehais, Raphaëlle N. Roy. Spectral EEG-based classification for operator dyads' workload and cooperation level estimation. IEEE SMC 2019, Oct 2019, Bari, Italy. pp.39153920. hal-02351808

\author{
HAL Id: hal-02351808 \\ https://hal.science/hal-02351808
}

Submitted on 6 Nov 2019

HAL is a multi-disciplinary open access archive for the deposit and dissemination of scientific research documents, whether they are published or not. The documents may come from teaching and research institutions in France or abroad, or from public or private research centers.
L'archive ouverte pluridisciplinaire HAL, est destinée au dépôt et à la diffusion de documents scientifiques de niveau recherche, publiés ou non, émanant des établissements d'enseignement et de recherche français ou étrangers, des laboratoires publics ou privés. 


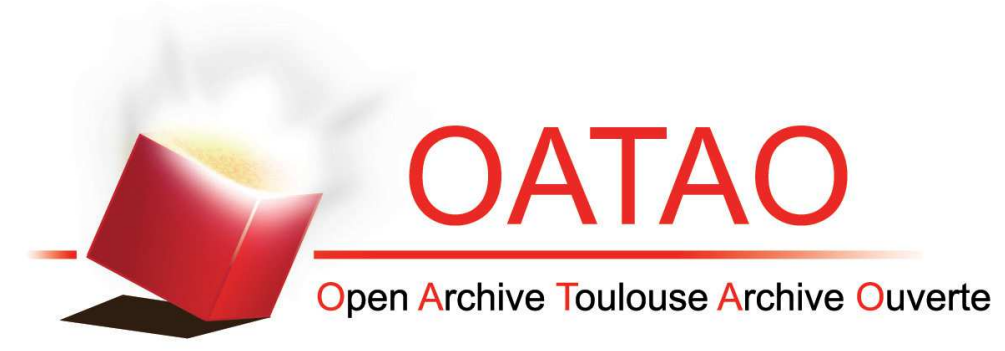

\section{Open Archive Toulouse Archive Ouverte (OATAO)}

OATAO is an open access repository that collects the work of some Toulouse researchers and makes it freely available over the web where possible.

This is an author's version published in: https://oatao.univ-toulouse.fr/24552

\section{To cite this version :}

Verdière, Kevin J. and Dehais, Frédéric and Roy, Raphaëlle N. Spectral EEG-based classification for operator dyads workload and cooperation level estimation. (2019) In: IEEE SMC 2019, 6 October 2019 - 9 October 2019 (Bari, Italy).

Any correspondence concerning this service should be sent to the repository administrator: tech-oatao@listes-diff.inp-toulouse.fr 


\title{
Spectral EEG-based classification for operator dyads' workload and cooperation level estimation
}

\author{
Kevin J. Verdière ${ }^{1}$, Frédéric Dehais ${ }^{1}$ and Raphaëlle N. Roy ${ }^{1}$
}

\begin{abstract}
There is a growing momentum to design online tools to measure mental workload for neuroergonomic purposes. Most of the research focuses on the monitoring of a single human operator. However, in real-life situations, human operators work in cooperation to optimize safety and performance. This is particularly the case in aviation whereby crews are composed of a pilot flying and a pilot monitoring. The motivation of this study is to evaluate the possibility to apply an hyperscanning approach to estimate the mental workload of crews composed of two operators. We designed an experimental protocol in which ten crews (i.e. 20 subjects) had to perform a modified version of the NASA MATBII during 8 five-minute blocks (i.e. 4 mental workload level configurations $* 2$ cooperation $\mathrm{v}$. non cooperation conditions). Mental workload and cooperation level were classified using a traditional passive brain-computer interface pipeline that includes a spatial filtering step on frequency features. Our results disclosed that all mental states' estimations were significantly above chance level. Intra-subject classification accuracy for mental workload ( 2 classes) was $63 \%$ for the pilot flying and $58 \%$ for the pilot monitoring. As for cooperation level, the binary classification reached $\mathbf{5 7 \%}$ for the pilot flying and $60 \%$ for the pilot monitoring. Regarding the team, intra-team classification accuracy of the workload configuration of the team (4-class) reached $35 \%$. As for the team cooperation level, the binary classifier reached $60 \%$ of accuracy. The results are discussed in terms of hyperscanning applications.
\end{abstract}

\section{INTRODUCTION}

Mental workload is a cognitive state that has been studied for decades. It is generally considered to be the result of the difficulty of a task and the associated effort furnished by the operator [1]. This mental state is of major interest to the human factor community as its impact on task performance has long been assessed. Classical ways of measuring mental states are subjective measures (i.e. questionnaires) and objective but indirect measures such as behavioral measures (i.e. reaction times and accuracy) or Autonomous Nervous System's measures (e.g. cardiac activity). Recently, researchers have been designing new means of performing a direct mental state assessment through cerebral measurements leading to the emergence of a field called Neuroergonomics, and thanks to machine learning techniques to a field called Physiological Computing and passive Brain-Computer Interfaces. Thus, cerebral activity measured using an electroencephalogram (EEG) has been shown to be significantly altered by an increasing cognitive workload. Indeed, an increase in the workload of an operator is linked to a decrease in

\footnotetext{
1 Kevin J. Verdière, Frédéric Dehais ${ }^{1}$ and Raphaëlle $\mathrm{N}$. Roy are with ISAE-SUPAERO, Université de Toulouse, France firstname. lastnamedisae.fr
}

the amplitude of temporal features called event-related potentials [2]-[4] and a power decrease in the alpha band $\left(\left[\begin{array}{ll}8 & 12\end{array}\right] \mathrm{Hz}\right)$ at centro-parietal sites jointly with an increase in power in the theta band ([4 7] Hz) at fronto-central sites [5]-[8].

Using dedicated classification pipelines on various cerebral measures several authors have been able to efficiently estimate an operator's mental workload in laboratory [3], [8], [9] or even in real life settings [10]-[12]. Despite their interest, most of these studies have focused on the monitoring of a single operator. However, one has to consider that human operators generally work in a cooperative fashion to achieve common goals. A typical example is the aviation domain whereby crews are composed of a pilot flying (PF), who is in charge of controlling the aircraft trajectory, and of a pilot monitoring (PM), who is in charge of monitoring the aircraft status and communicating with air traffic controller [13], [14]. Hence, there is a need to implement monitoring solutions at the team level as episodes of high workload have been shown to disrupt cooperation [15], [16].

A recent field of research has tried to address this issue by identifying physiological proxies of cooperation over time. For instance, the physiological substrates of cooperation, also called 'physiological compliance', have been studied using protocols that involve teams of 2 (dyads) or more participants (for a review see [17]). Since the first 'hyperscan' (multiple brain recording [18]) some studies were performed in the field of game theory, decision making or cooperative $\mathrm{v}$. competitive behavior. Yet only a few of them did actually relate to team workload (for a review on hyperscanning in Social Neuroscience see [19]). For example, Stevens and collaborators [20] presented a quantitative model based on entropy measures to assess teams' neurodynamics during complex cooperative task performance (i.e. submarine piloting task or healthcare training). Also, Toppi and collaborators [21] found significant differences in graph metrics computed from Partial Directed Coherence in PF-PM teams during simulated flights. Lastly, Korczowski and collaborators [22] demonstrated the performance of Riemannian geometry for classification of Multi-User P300-Based Brain-Computer Interface.

However none of these two studies tried to classify and estimate operators' state using a passive Brain-Computer Interface (BCI) pipeline. This a very recent field of study and to 
our knowledge the efficient estimation of a team's workload through cerebral measures has not yet been published, and nor is it the case for a team's cooperation level. To meet this challenging goal, couples of participants had to perform a modified version of the NASA MATB-II in which we manipulated the levels of mental workload and cooperation. This experimental set-up aimed at reproducing the flying operational context as participants played either the role of a PF or a PM. With this protocol we hypothesized that, because mental workload and cooperation elicit different behaviors and different neurodynamics, [19]-[21], a classifier based on spectral EEG features should be able to discriminate those mental states. To evaluate this hypothesis, mental workload and cooperation levels were classified using a traditional passive BCI pipeline that includes a spatial filtering step on frequency features.

\section{METHODS}

\section{A. Participants}

Twenty participants (i.e. 10 teams; 4 females; 25 years old in average \pm 7 ) underwent this experiment on a volunteer basis. They were recruited among the students of the ISAE-SUPAERO engineering school. All had normal or corrected-to-normal vision and no history of neurological or psychiatric disorders. The study was approved by the local ethic committee (IRB00011835-2019-05-28-1) and all participants gave their informed written consent.

\section{B. Experimental Protocol}

The participants took part in an experiment that included a modified version of the MATB micro-world [23]. Each couple performed 8 scenarios of 5 minutes each. During the whole experiment each participant was either Pilot Flying (PF) or Pilot Monitoring (PM), a role attributed randomly by the experimenter. The PF had to perform the System Monitoring (Sysmon) task using the keyboard along with the Tracking (Track) task using a joystick. The PM had to manage the Communications (Comm) with the mouse along with the Resource Management (Resman) Task using the numerical keyboard. Two levels of difficulty were modulated separately for each participant (i.e. Easy and Hard) which resulted in two mental workload conditions (i.e. Low and High). For the PF, only the Track difficulty was modulated by lowering its responsiveness and increasing its speed. For the PM, only the Resman task difficulty was modulated by increasing the flowrates and increasing the number and the duration of failed pump. Moreover, the participants were also asked either to cross-monitor and help their partner (cooperative condition) or not (control condition). Cross-monitoring refers to interacting directly with the partner' tasks without speaking (i.e. doing his/her task). Those modulations resulted in a total of 8 possible conditions (i.e. 8 blocks). All the conditions/blocks were pseudo-randomly presented.

\section{Data Acquisition}

The participants' subjective reports of workload were recorded thanks to the NASA TLX questionnaire [24]. Also, their performance was computed thanks to a score based on their performance to each of the MATB sub-tasks. Moreover, the participants' EEG were recorded at $256 \mathrm{~Hz}$ with two BioSemi ActiveTwo 64 active electrodes positioned according to the 10-20 system. The Common Mode Sense (CMS) active electrode and the Driven Right Leg (DRL) passive electrode were positioned next to POz. Data from the task and both EEG systems were synchronized and recorded with the help of Lab Streaming Layer [25].

\section{Classification Procedure}

Classifiers trained on frequency features (theta, alpha and low beta power) were used to detect if we could discriminate the workload level for each participant (binary), and the workload configuration for each team (i.e. low/low, low/high, high/low or high/high, hence 4-class). In the same manner classifiers were trained to detect the cooperation level (always binary) for each subject, and for the team. To do so, a classification pipeline close to that used by Dehais and collaborators [12] and by Roy and collaborators [8] was used. The EEG data processing and classification were run using EEGLab (V14.1.2b) and Matlab (R2019a).

First, every block was cut into 2-sec epochs. Each epoch was preprocessed independently in order to potentially extend our method to online processing. The data were high-pass filtered $(0.5 \mathrm{~Hz})$ and noisy portions were removed using the ASR plugin of the toolbox available at [26]. Sources of noises were mainly due to movement and physiological noises (eg. Eye movement, cardiac activity). Next, for each epoch the frequency power in different frequency bands (theta [4 8] Hz, alpha [8 12] Hz and low beta $[1216] \mathrm{Hz}$ ) was computed. To compute these frequency power features, the EEG signals were filtered in each band using a 150-order FIR filter. Then, a Common Spatial Filter (CSP) was computed for each band and only 4 filters were kept. The features then consisted of the log variance of the spectrally and spatially filtered signal. In total there were 12 features per epoch ( 3 frequency bands*4 filters).

Lastly, a subject-dependent 2-class or 4-class (depending on the study) shrinkage Linear Discriminant Analysis (sLDA) classifier was used, as recommended in [27], with a One-Versus-The-Rest (OVR) strategy for the 4-class. A 4-Fold Cross Validation procedure was applied by keeping one block (scenarios) of each class out per fold. All classifiers were trained using data from blocks different from the ones used for testing. The theoretical chance threshold was adjusted following the recommendations of [28]. 

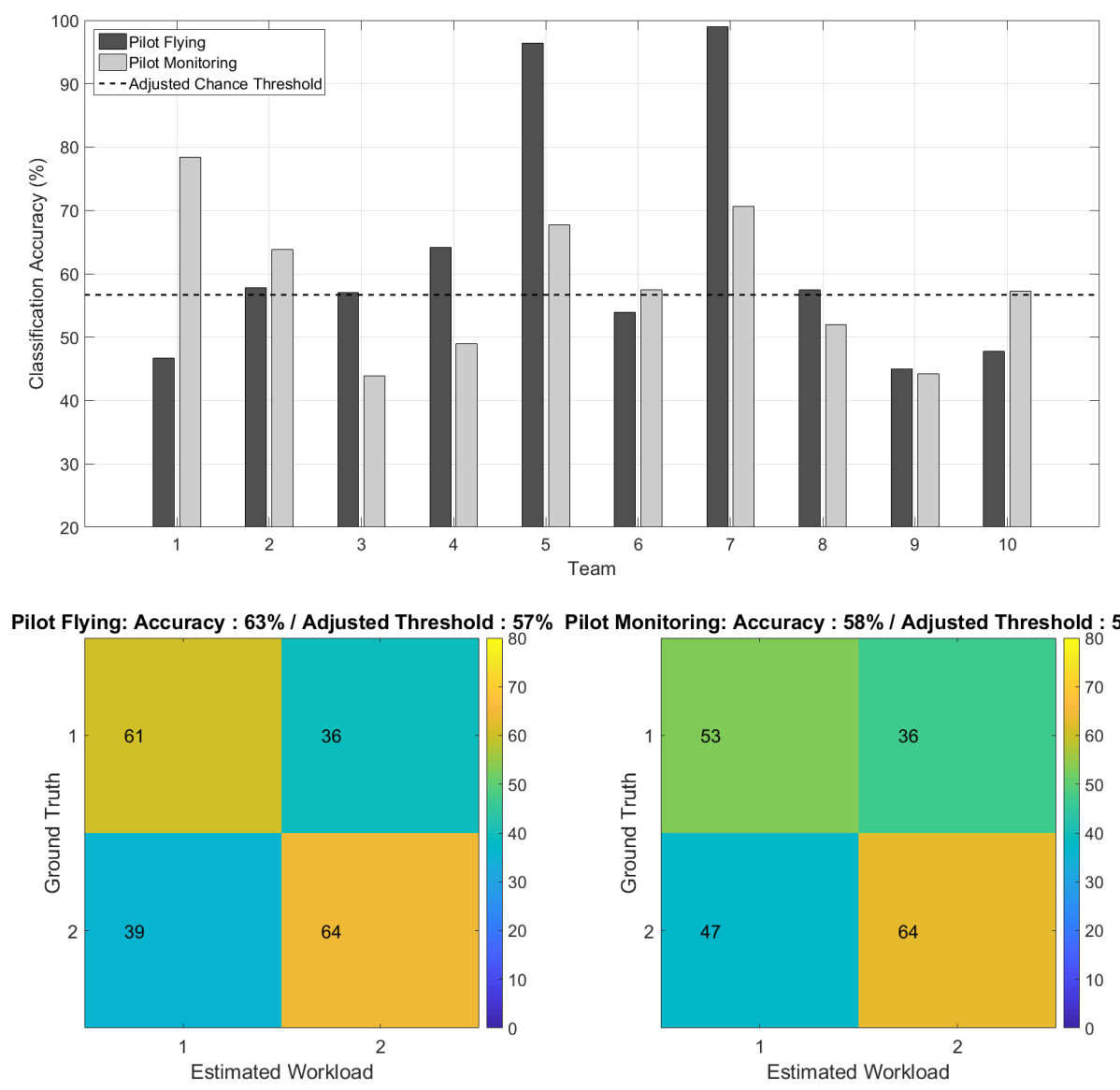

Fig. 1. Upper : Binary workload classification performance from a single operator's EEG data. Lower : Confusion matrices for the binary workload classification performance from a single operator's EEG data

\section{E. Statistical Analyses}

The subjective, behavioral, EEG and classification results were all statistically analyzed using the Statistica software. A repeated measures ANOVA with workload for PF and PM, cooperation and electrodes (WK PF x WK PM x COOP x ELECT) was used. Tukey HSD was applied to post-hoc tests to correct for multiple comparisons. For the sake of clarity and to facilitate the reading of the results only the data of 4 electrodes frequently cited in the literature were analyzed for the physiological analyses, namely $\mathrm{Fz}, \mathrm{Cz}, \mathrm{Pz}$ and $\mathrm{Oz}$. Yet all 64 electrodes were used for the classification step.

\section{RESULTS}

\section{A. Protocol validation}

In order to validate the experimental protocol designed to modulate mental workload, we verified whether the participants did indeed feel a higher strain in the high load condition as compared to the low one, and whether their performance was decreased by an increase of workload.

1) Subjective Reports: As regards the subjective reports of workload measured thanks to the NASA TLX, in a general manner the operators who performed the task as pilot monitoring reported a higher workload when they underwent the task in the high difficulty condition (PM Difficulty) than in the low one $(\mathrm{F}(1,9)=3062.8, p<0.01)$. In the same manner, the operators that performed the task as pilot flying reported a higher workload when they underwent the task in the high difficulty condition (PF Difficulty) than in the low one $(F(1,9)=29.32, p<0.01)$. There was no significant differences in the subjective ratings regarding the cooperation condition.

2) Behavioral Data: Regarding the behavioral data, the operators' performance dropped when workload increased for the pilot flying $(\mathrm{F}(1,9)=5.21, p<0.05)$, as well as when it increased for the pilot monitoring $(\mathrm{F}(1,9)=5.22, p<$ $0.05)$. There was no significant differences in performance regarding the cooperation condition.

\section{B. EEG Data}

The power of the three frequency bands of interest -theta, alpha and low beta- was modulated by both workload and cooperation. Indeed, the low beta power of the PF decreased significantly at $\mathrm{Fz}$ in the cooperation condition when PM had a high workload $(p<0.05)$. The low beta power of the PM significantly increased with an increase in workload of the PF $(p<0.05)$ and significantly decreased with an 
increase in workload of the PM herself $(p<0.01)$.

Alpha power of the PM significantly decreased at all sites with an increase in workload level of the PM in the control condition $(p<0.05)$; it also significantly decreased with cooperation when workload was low for the PM and high for the PF $(p<0.05)$. As for the PF, there was only a trend of increase in alpha power at $\mathrm{Pz}$ with increasing workload and cooperation $(p=0.08)$. This trend was mirrored by a trend for a decrease in theta power at all sites with increasing workload and cooperation $(p=0.08)$. Lastly, there was a trend for a decrease in theta power of the PM when the load of the PF increased ( $p=0.07$ ); there was also a trend for a decrease in theta power in the cooperation condition compared to the control condition $(p=0.06)$.

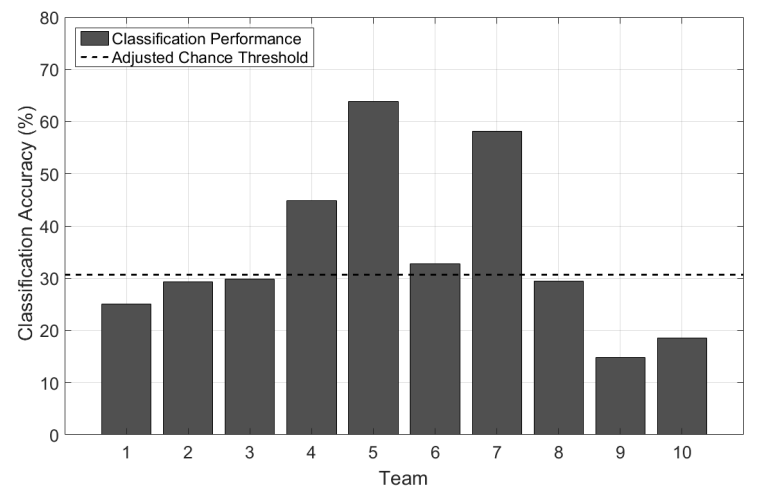

2 operators: Accuracy : $35 \%$ / Adjusted Threshold : $31 \%$

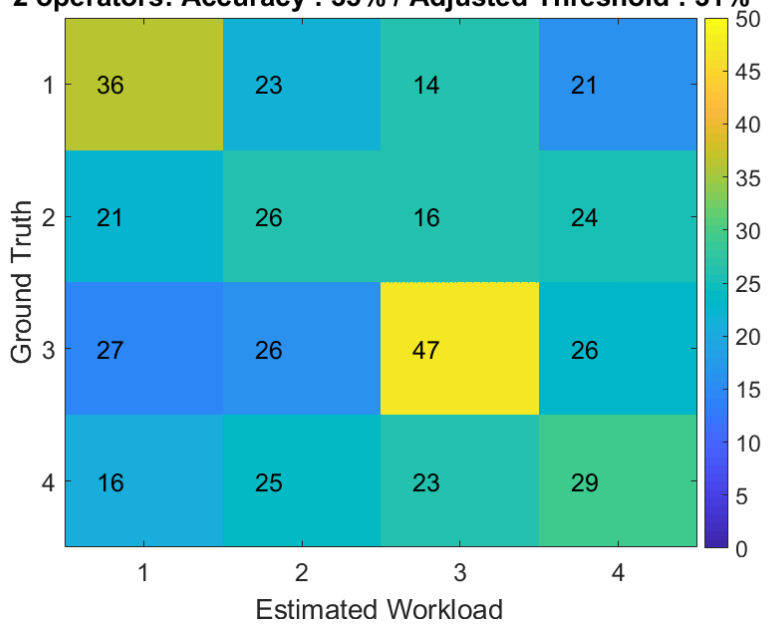

Fig. 2. Upper : 4-class workload/configuration classification performance from two operators' EEG data. Lower : Confusion matrix for the 4-class workload classification performance from both operators' EEG data

\section{Classification Results}

All classification results were significantly above the adjusted chance level. The workload condition of each operator was estimated in an intra-subject fashion using the pipeline described earlier. As illustrated by Figures 1, the binary classification reached $63 \%$ of accuracy for the pilot flying and 58\% for the pilot monitoring (adjusted threshold: $57 \%$ ). Also, using both operators the workload configuration (i.e. low/low, high/low, low/high or high/high) could be estimated with a 4-class classifier with an accuracy of $35 \%$ (adjusted threshold: 31\%; Figures 2).

Regarding cooperation level estimation, the binary classification reached $57 \%$ using for the pilot flying, $60 \%$ using the pilot monitoring and $60 \%$ using the team (adjusted threshold: 57\%; Figures 3 and 4). There was a trend for a better cooperation estimation using both operators' features than only the PF ones $(p=0.07)$.

\section{DISCUSSION \& CONCLUSION}

The main goal of this study was to assess whether workload and cooperation level could be estimated using typical EEG features (i.e. spectral features: power in the theta, alpha and beta bands) and a classification pipeline using data from one single operator or the whole dyad. First, the protocol that was used did manipulate the workload of both operators as demonstrated by statistically significant subjective, behavioral and physiological modulations.

Next, it appeared that indeed both mental states could be estimated from the EEG spectral features of each operator with classification accuracies above the adjusted chance level with as high as $63 \%$ of accuracy for workload binary estimation which is inline with previous findings [8]. Cooperation level was also classified as high as $60 \%$ independently of the workload level. Yet to our knowledge there is no literature to compare this result to. Interestingly there was not much benefit of using both operators' features since the classification performance of using one operator's features and using both operators' were not statistically different. However in a hyperscanning framework it might be relevant to assess operators' mental states using both single operators' features and dyadic features to ensure a correct decision on a given team's state by the automated system.

Interestingly enough there was a slight difference in workload estimation depending on the role (i.e. PF or PM). Indeed the workload estimation dropped to $58 \%$ on the PM. Her/his tasks might not elicit a workload level as high as the PF's one. This might also due to the fact that the model was trained as if there was only 2 conditions for a participant: Easy and Hard. The reality was that the workload of one participant (PF or PM) could be greatly influenced by the performance of the partner as the task was developed to have dependencies among subs-tasks. For example, while a scenario equated as an Easy one for a PM, if the PF performed poorly at the tracking task, this easy scenario could have been even harder than a hard one for the PM. This strong dependency, especially from the track to the resource management might be a reason for this accuracy gap. When evaluating a team member workload, it might be irrelevant to look only at one teammate difficulty regardless of their partners difficulty. Yet this implementation allowed 


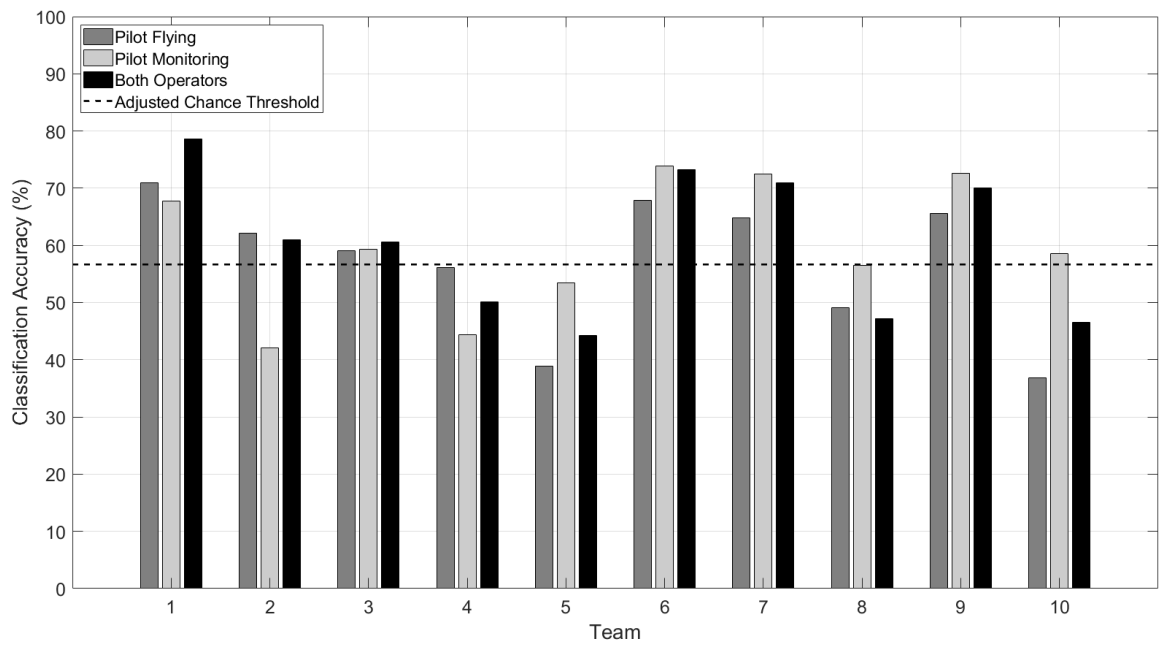

Fig. 3. Binary cooperation level classification performance from each type of operator or both operators' EEG data
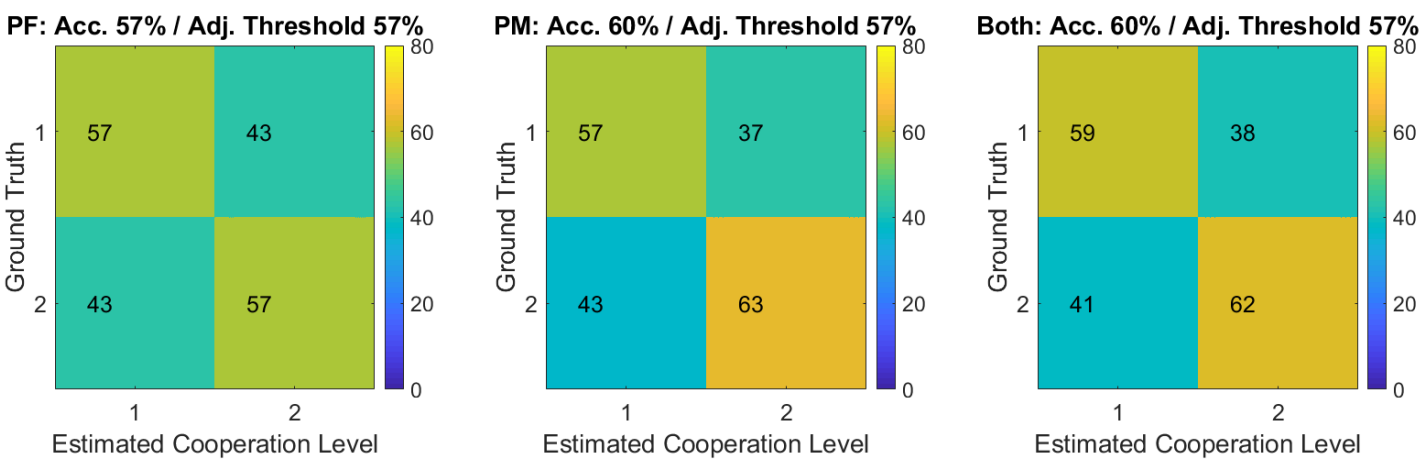

Fig. 4. Confusion matrices for the binary cooperation level classification performance from each type of operator or both operators' EEG data

to work on the basis of a close to real-life task.

This study showed that it is possible to detect workload level and cooperative states using spectral EEG features from a 2 -second window. The results compare to classifier performance obtained with a fatigue inducing protocol [29] where $65 \%$ were achieved. Moreover classifiers were tested with epochs from scenarii they were not trained on. This prevented us to spuriously increase the classification rate by detecting spectral similarities in contiguous epochs. Yet improvements still remain to be done in order to greatly increase the classification performance. For instance, the classification pipeline that was used was applied at the sensor level. It would be interesting to extend the analysis to the source level by using ICA [30] or LORETA [31]. Also, a spatial filter was used to summarize spectral information from the electrodes. Instead, connectivity metrics could be used as features as previously demonstrated for mental state monitoring purposes on either a single operator or on dyads [11], [21], [32], [33]. In addition, although 10 couples were used, classification was done at the subject or team level. Another solution could be to perform intersubject or interteam prediction using linear regression methods [34].
Further, practical limitations of this protocol (i.e. temporal constraints) compelled subjects to realize only one block per condition. Hence classification could not be performed over a single condition. Epochs were either classified as low or high regardless of the partner's task difficulty or the cooperative state. Therefore this might not reflect very accurately the actual workload experienced by the participants and more generally their mental states. Therefore, it would be useful to implement a more refined protocol considering levels of workload and with more repetitions in order to classify the exact same conditions. Moreover, this pipeline should be evaluated with a portable EEG system to get closer to ecological conditions. While this protocol open the way to real world setting condition, the practical application will still remain a great challenge.

Conversely while cooperation was also classified regardless of the workload level, it seems even more interesting that the classification level reached $60 \%$. This results means that despite opposite levels of workload, cooperative behavior might elicit specific detectable and robust spectral EEG patterns. However one has to note that 
the protocol asks participants to cooperate but they were not required to do so. In other words, the classification could have been performed over the actual observed level of cooperation instead of the cooperative instructions.

To conclude, to our knowledge this study is the first one to try and assess dyadic mental workload and cooperation level using EEG spectral features and a classification pipeline. Hyperscanning is a promising tool for performance and security improvements in risky work settings and this promising study paves the way for mental state monitoring applications on teams of operators.

\section{ACKNOWLEDGMENT}

This study was supported by a PhD grant delivered by the DGA (Direction Générale de l'Armement).

\section{REFERENCES}

[1] A. Gevins and M. E. Smith, "Electroencephalography (eeg) in neuroergonomics," Neuroergonomics: The brain at work, pp. 15-31, 2006.

[2] B. Z. Allison and J. Polich, "Workload assessment of computer gaming using a single-stimulus event-related potential paradigm," Biological psychology, vol. 77, no. 3, pp. 277-283, 2008.

[3] R. N. Roy, S. Charbonnier, A. Campagne, and S. Bonnet, "Efficient mental workload estimation using task-independent EEG features," Journal of neural engineering, vol. 13, no. 2, p. 026019, 2016.

[4] A.-M. Brouwer, M. A. Hogervorst, J. B. Van Erp, T. Heffelaar, P. H. Zimmerman, and R. Oostenveld, "Estimating workload using eeg spectral power and erps in the n-back task," Journal of neural engineering, vol. 9, no. 4, p. 045008, 2012.

[5] A. Stipacek, R. Grabner, C. Neuper, A. Fink, and A. Neubauer, "Sensitivity of human eeg alpha band desynchronization to different working memory components and increasing levels of memory load," Neuroscience letters, vol. 353, no. 3, pp. 193-196, 2003.

[6] P. Missonnier, M.-P. Deiber, G. Gold, P. Millet, M. G.-F. Pun, L. FazioCosta, P. Giannakopoulos, and V. Ibáñez, "Frontal theta event-related synchronization: comparison of directed attention and working memory load effects," Journal of Neural Transmission, vol. 113, no. 10, pp. 1477-1486, 2006.

[7] P. Antonenko, F. Paas, R. Grabner, and T. Van Gog, "Using electroencephalography to measure cognitive load," Educational Psychology Review, vol. 22, no. 4, pp. 425-438, 2010.

[8] R. N. Roy, S. Bonnet, S. Charbonnier, and A. Campagne, "Mental fatigue and working memory load estimation: interaction and implications for eeg-based passive bci," in 2013 35th annual international conference of the IEEE Engineering in Medicine and Biology Society $(E M B C)$. IEEE, 2013, pp. 6607-6610.

[9] G. Durantin, J.-F. Gagnon, S. Tremblay, and F. Dehais, "Using near infrared spectroscopy and heart rate variability to detect mental overload," Behavioural brain research, vol. 259, pp. 16-23, 2014.

[10] T. Gateau, H. Ayaz, and F. Dehais, "In silico versus over the clouds: On-the-fly mental state estimation of aircraft pilots, using a functional near infrared spectroscopy based passive-bci," Frontiers in human neuroscience, vol. 12, p. 187, 2018.

[11] K. J. Verdière, R. N. Roy, and F. Dehais, "Detecting pilot's engagement using fnirs connectivity features in an automated vs. manual landing scenario," Frontiers in human neuroscience, vol. 12, p. 6, 2018.

[12] F. Dehais, A. Duprès, S. Blum, N. Drougard, S. Scannella, R. N. Roy, and F. Lotte, "Monitoring pilots mental workload using ERPs and spectral power with a six-dry-electrode EEG system in real flight conditions," Sensors, vol. 19, no. 6, p. 1324, 2019.

[13] C. A. Authority, "Monitoring matters, guidance on the development of pilot monitoring skills," CAA Paper, vol. 2, 2013.

[14] I. International Air Transport Association, "Guidance material for improving flight crew monitoring," IATA Paper, 2016.

[15] F. Dehais, J. Behrend, V. Peysakhovich, M. Causse, and C. D. Wickens, "Pilot flying and pilot monitorings aircraft state awareness during go-around execution in aviation: A behavioral and eye tracking study," The International Journal of Aerospace Psychology, vol. 27, no. 1-2, pp. 15-28, 2017.
[16] M. Reynal, Y. Colineaux, A. Vernay, and F. Dehais, "Pilot flying vs. pilot monitoring during the approach phase: An eye-tracking study," in Proceedings of the International Conference on Human-Computer Interaction in Aerospace. ACM, 2016, p. 7.

[17] R. V. Palumbo, M. E. Marraccini, L. L. Weyandt, O. Wilder-Smith, H. A. McGee, S. Liu, and M. S. Goodwin, "Interpersonal autonomic physiology: A systematic review of the literature," Personality and Social Psychology Review, vol. 21, no. 2, pp. 99-141, 2017.

[18] P. R. Montague, G. S. Berns, J. D. Cohen, S. M. McClure, G. Pagnoni, M. Dhamala, M. C. Wiest, I. Karpov, R. D. King, N. Apple et al., "Hyperscanning: simultaneous fmri during linked social interactions," 2002.

[19] F. Babiloni and L. Astolfi, "Social neuroscience and hyperscanning techniques: past, present and future," Neuroscience \& Biobehavioral Reviews, vol. 44, pp. 76-93, 2014.

[20] R. H. Stevens, T. L. Galloway, and A. Willemsen-Dunlap, "Neuroergonomics: Quantitative modeling of individual, shared, and team neurodynamic information," Human factors, vol. 60, no. 7, pp. 10221034,2018

[21] J. Toppi, G. Borghini, M. Petti, E. J. He, V. De Giusti, B. He, L. Astolfi, and F. Babiloni, "Investigating cooperative behavior in ecological settings: an eeg hyperscanning study," PloS one, vol. 11, no. 4, p. e0154236, 2016.

[22] L. Korczowski, M. Congedo, and C. Jutten, "Single-trial classification of multi-user p300-based brain-computer interface using riemannian geometry," in 2015 37th annual international conference of the IEEE engineering in medicine and biology society $(E M B C)$. IEEE, 2015, pp. 1769-1772.

[23] J. R. Comstock Jr and R. J. Arnegard, "The multi-attribute task battery for human operator workload and strategic behavior research," 1992.

[24] S. G. Hart and L. E. Staveland, "Development of nasa-tlx (task load index): Results of empirical and theoretical research," in Advances in psychology. Elsevier, 1988, vol. 52, pp. 139-183.

[25] C. Kothe, "Lab streaming layer (ls1)," https://github. com/sccn/labstreaminglayer. Accessed on October, vol. 26, p. 2015,2014

[26] T. Mullen, C. Kothe, Y. M. Chi, A. Ojeda, T. Kerth, S. Makeig, G. Cauwenberghs, and T.-P. Jung, "Real-time modeling and 3d visualization of source dynamics and connectivity using wearable eeg," in 2013 35th annual international conference of the IEEE engineering in medicine and biology society (EMBC). IEEE, 2013, pp. 2184-2187.

[27] F. Lotte, L. Bougrain, A. Cichocki, M. Clerc, M. Congedo, A. Rakotomamonjy, and F. Yger, "A review of classification algorithms for eeg-based brain-computer interfaces: a 10 year update," Journal of neural engineering, vol. 15, no. 3, p. 031005, 2018.

[28] E. Combrisson and K. Jerbi, "Exceeding chance level by chance: The caveat of theoretical chance levels in brain signal classification and statistical assessment of decoding accuracy," Journal of neuroscience methods, vol. 250, pp. 126-136, 2015.

[29] R. N. Roy, S. Bonnet, S. Charbonnier, and A. Campagne, "Mental fatigue and working memory load estimation: interaction and implications for eeg-based passive bci," in 2013 35th annual international conference of the IEEE Engineering in Medicine and Biology Society $(E M B C)$. IEEE, 2013, pp. 6607-6610.

[30] S. Makeig, A. J. Bell, T.-P. Jung, and T. J. Sejnowski, "Independent component analysis of electroencephalographic data," in Advances in neural information processing systems, 1996, pp. 145-151.

[31] R. D. Pascual-Marqui, M. Esslen, K. Kochi, D. Lehmann et al., "Functional imaging with low-resolution brain electromagnetic tomography (loreta): a review," Methods and findings in experimental and clinical pharmacology, vol. 24, no. Suppl C, pp. 91-95, 2002.

[32] S. Charbonnier, R. N. Roy, R. Doležalová, A. Campagne, and S. Bonnet, "Estimation of working memory load using EEG connectivity measures," in Proceedings of the 9th International Joint Conference on Biomedical Engineering Systems and Technologies - Volume 4: BIOSIGNALS, (BIOSTEC 2016). SciTePress, 2016, pp. 122-128.

[33] S. Charbonnier, R. N. Roy, S. Bonnet, and A. Campagne, "EEG index for control operators mental fatigue monitoring using interactions between brain regions," Expert Systems with Applications, vol. 52, pp. 91-98, 2016.

[34] K.-J. Chiang, C.-S. Wei, M. Nakanishi, and T.-P. Jung, "Cross-subject transfer learning on high-speed steady-state visual evoked potentialbased brain-computer interface," arXiv preprint arXiv:1810.02842, 2018. 